\title{
A Novel Wireless Intercommunication Device for Real Time Applications
}

\author{
Shivaraman Ilango \\ Heath care Technology and innovation center
}

\begin{abstract}
Indian Institute of Technology, Madras Research Park Abstract: The present invention relates to communication using wireless technology through free band (commonly called citizen band) and licensed band in various establishments in particular, where the wireless technology is employed in the form of a device integrated on any person or on any moving object through radio frequency waves. It is a profound full duplex communication without the use of Satellite or GSM technologies.
\end{abstract}

Keywords: Base station, Dial pads, Full duplex, Radio Frequency, Transceivers,

\section{Introduction}

With existing technology communication within an organization is done using intercom. Here the intercom lines are fixed to a particular place in a room, preferably the table of the person using it. This uses long running chords to connect the various users through a telephone. It involves a costly infrastructure outlay and maintenance. These cannot be carried along with the person when he travels out of the room/ area in which the intercom is installed. It is immobile. Further, it cannot be used if there is damage to the connecting chord, which might be for various reasons including due to fire, natural calamity or any other disaster. In some establishments such as the police department or the security agencies communication is done using wireless communication technology using walkie talkies, where the data and voice signals can be transmitted and received by transceiver circuits using RF free band, which achieves only half duplex. Further, the frequency range is limited to less than $2.4 \mathrm{Gega}$ Hertz. This is without the use of GSM and blue tooth devices. Here there is a more flexible frequency range that is possible and makes long distance communication highly effective.

Further, in communication using the above, data and voice signals are transmitted and received by transceiver circuits in RF free band. Here the dialer initiates the communication by pressing a push button which sends an RF signal to the control room, which in turn authorizes the signal to be transmitted to all other walkie talkies connected on that particular frequency, within that area. This is through satellite communication. Further, the person wishing to send a voice signal has to continuously press and hold the push button while talking. Once he releases the button, the transmitting circuit is closed and the voice communication is cut off. The drawback of this is that full duplex is not possible, continuous holding of the push button during talking is required apart from requiring the assistance of a satellite.

\section{Description Of Invention}

The present invention is wirelesses inter communication network device comprising of two essential parts namely, multiple dial pads and a base unit for data and voice communication through wireless.

The multiple dial pads are interlinked to each other through a base unit through radio frequency waves or shock waves. The base unit is stationary and kept in a fixed place. The dial pad which is a communication device is small and compact hand set which is portable and can thus be carried by a person in his pocket or fitted on any moving or stationary object. The dial pad operates within a fixed distance from the base unit, which is determined based on the user requirements and legal permissible limits.

The radio frequency wave or shock wave are generated by the dial pad and is used to transmit the data and voice signal to the other dial pad/ pads, which receive the signal, through the base unit, which acts as an interlink for simultaneous two way communication between two or more dial pads. In this way, two way communication is achieved through wireless using this wave, by this wireless communicating network device. The frequency of the radio frequency waves or the shock waves generated is in the human audible frequency range i..e. from 3 to $30,000 \mathrm{~Hz}$. Further, depending upon the distance within which the communication is to be effected between these dial pads, the strength of the carrier wave is altered.

\section{Example 1}

For communicating within a 50 meter radius each dial pad should be within a distance of 50 meter from the base unit. The frequency of the radio frequency wave or shock wave is between 313 to $318 \mathrm{MHz}$ or........respectively. The power required is 230 volts. With this device, effective intercommunication between various personnel of a huge industrial, business organization or establishment, Government Departments, 
Offices, etc spread over a vast area is possible through wireless. This can also be used by security agencies, educational institutions, hospitals, Court Houses and various other organizations establishments where quick and effective communication is required among the various persons regarding the day to day operations/ functions, within the campus.

Dial Pad

Each dial pad is a compact and small communication device such as a hand set, consisting of transmitting antenna, transreceiver, push button, micro controller, power amplifier, crystal oscillator, audio speaker and mike connected to an in built power source. Each component is explainer below briefly.

\section{Base Unit}

The base unit is an electric and electronic circuit consisting of various components such as Transceivers, receiving antenna, micro controller, relay circuit connected in series, mike and speaker power amplifier, radio frequency digitalized tuner all connected to a power source

All these components in the base unit receive data and voice signals from one or more dial pads and transmit it to the other dial pad/ pads. This is achieved by switching a realy. Because of this simultaneous full duplex voice communication is achieved between two or more dial pads.

The radio frequency waves or shock waves generated from the dial pad are frequency modulated to ensure quality of voice communication.

\section{Working Of Invention}

This wireless communication network device comprises of multiple dial pads interlinked to each other through a static base unit using radio frequency or shock waves. When a bush button is pressed in the dial pad, the data that is programmed in the micro controller, relating to push button pressed is transmitted through the transmitting antenna to the base unit. The base unit consists of a receiving antenna, that receives and decodes these signals and sees which data that is programmed in the microcontroller present in the base unit is matched to data that is transmitted by the transmitting antenna. According to the received data it connects or calls that particular communication device through the use of relay circuits that switches the call to the device to be connected. The dial pad consists of an in built speaker and microphone unit for voice communication. Here a power amplifier can be used to alter the strength of the carrier signals/waves, so that it travels a long distance. Once the dial pad/communication device is connected, the data transfer is cut off and voice communication starts to commence between the two dial pads. This voice communication here is a full duplex/two way communication. This full duplex voice communication is achieved through the use of transceivers, present both in the dial pad/communicating device and the base unit. The transceiver circuit consists of a radio frequency digitalized tuner to transfer voice communication at particular frequency that is tuned to match in both dial pad and base unit. Mike and Speaker pre amplifier would be present to reduce or avoid any distortion during voice communication. The Radio frequency waves/ shock waves are transmitted and received by the transceivers present both in dial pad and base unit. If both wave frequencies are matched, then the voice communication takes place.

Both the base unit and dial pad are powered using an external power source and in built power source respectively.

The dial pad can also be connected to a hearing aid with a mike through wireless or through cord for the purpose of working convenience.
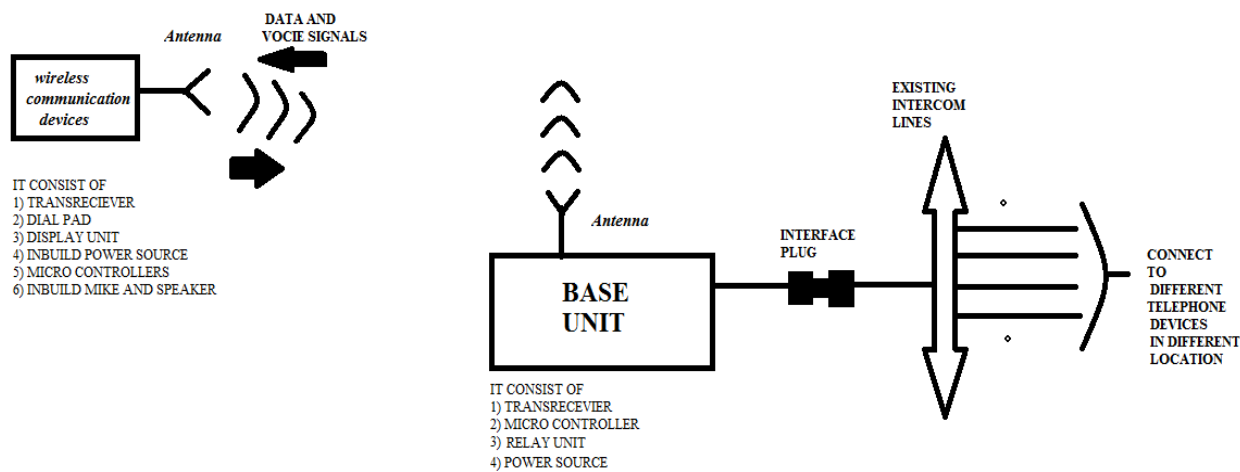

Fig 1 
The above figure makes the flexibility and portability of the base unit. This base unit can be taken anywhere within an industry or an organization plugged in to the existing intercom ports and patched up with the main distribution frame and communicate with the existing telephones anywhere within the industry. This will help in saving resources and cost for the organization.
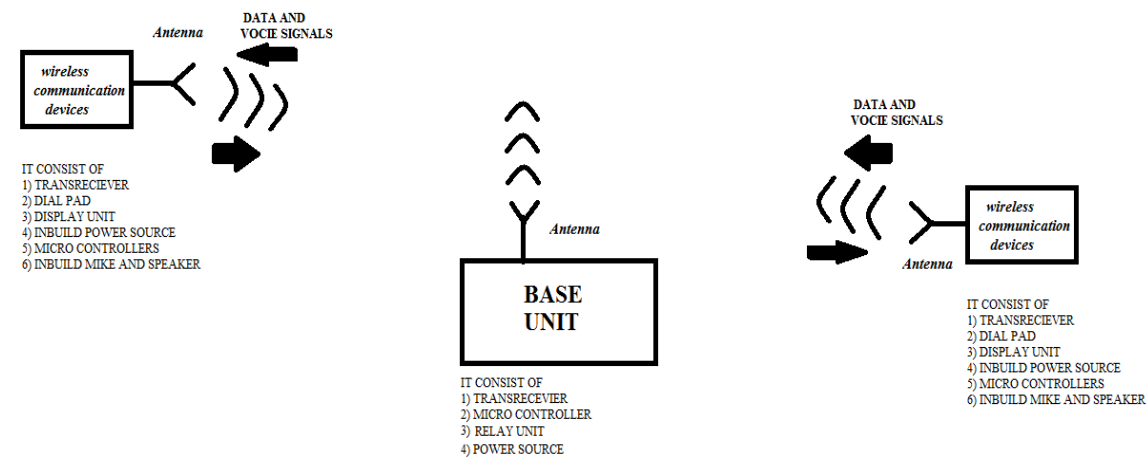

Fig 2

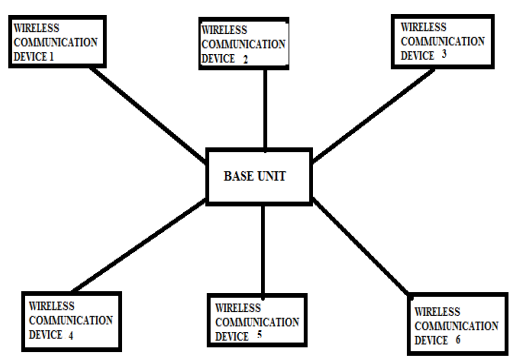

Fig 3

The above two diagrams show a hand held device that can be used for communicating with another hand held device and multiple hand held devices through the same radio frequency protocol

The advantages of the present invention:

\section{Advantages}

1. It is highly cost effect,

2. No charges is incurred for the calls

3. Calls cannot be tapped, hacked

4. Use of satellite is restricted, avoided.

5. Portable and handy.

6. Used for diagnostic work also.

7. Avoids the use of blue tooth, SIM card or other GSM techniques.

\section{Conclusion}

This small portable communication device gives a new dimension to the other handheld communication devices and can be used in all real time scenarios. Based on the present invention using one dial pad, a person in an organization campus can communication with various others within the campus who are situated at other locations, quickly, effectively and conveniently through wireless. This is very effective in cases of accidents, emergencies, natural disasters and for efficient functioning, when the conventional modes of chord connected intercoms or telephones fails for various reasons in various situations, in particular the above mentioned situations

\section{Acknowldgement}

This Project was sponsored by BHAVINI (India's first prototype fast breeder reactor) and the invention is to be implemented in their organization. 


\section{References}

[1] Drives and Controls (2000). PLC, phone home.October issue.

[2] Hogan, R. J. (2003). Wireless expansion and enhancements of ICAS.NAVSEA 13th international ship control systems symposium 2003, Orlando, USA

[3] Harman, R., \& Clark, D. (2003)."Wireless machinery monitoringsystems for shipboard and power plant applications". NAVSEA 13th international ship control systems symposium 2003, Orlando, USA

[4] Maes, M. Mhoon, D., Currie, D., \& Ralston, J. (1999). "Extend your plant network with industrial wireless Ethernet". I\&CS, September issue.

[5] Daniel, R. J., Estes, A., Sarkady, A., Welch, T. B., \&Whitese, H.(2003). Assessment of radio frequency propagation in naval shipboard environment with Bluetooth applications. NAVSEA 13th international ship control systems symposium 2003, Orlando,USA

[6] Jakes WC. Microwave mobile communication. 2nd ed., New York: IEEE Press; 1994.

[7] Kamio Y, Sampei S, Sasaoka S, Morinaga N. Performance of modulation-level-controlled adaptive-modulation under limited transmission delay time for land mobile communications. In: Proceedings of the IEEE veh technol conf (VTC95), Chicago, Illinois, 1995, p. 221-5. 